\title{
Performance of three transducer mounting methods in impedance-based structural health monitoring applications
}

Journal of Intelligent Material Systems and Structures

20I7, Vol. 28(I7) 2349-2362

(C) The Author(s) 2017

Reprints and permissions:

sagepub.co.uk/journalsPermissions.nav DOI: I0.II77//045389XI7689942

journals.sagepub.com/home/jim (S)AGE

\author{
Ricardo Zanni Mendes da Silveira, Leandro Melo Campeiro and \\ Fabricio Guimarães Baptista
}

\begin{abstract}
Piezoelectric transducers are widely used in many nondestructive methods for damage detection in structural health monitoring applications. Among the various methods for detecting damage, the electromechanical impedance technique is known for using thin and small piezoelectric ceramics operating simultaneously as actuators and sensors. The basic method of installing these piezoelectric ceramics in the host structure is using a high-stiffness adhesive such as epoxy or cyanoacrylate glue. However, some studies have proposed alternative methods of transducer mounting, therein aiming to reuse the transducer or allowing for the monitoring of structures under adverse conditions under which the direct installation of the sensor would not be possible. Thus, the objective of this study is to analyze and compare the performance of three main mounting methods for metal structures for applications based on the electromechanical impedance technique: magnetic mounting, metal-wire-based mounting, and conventional mounting using adhesives. Tests were conducted on aluminum beams, and the performances of the three transducer mounting methods were compared using basic damage indices and the pencil-lead-break test. The experimental results indicate that the mounting method has a significant effect on the frequency response and sensitivity for damage detection.
\end{abstract}

\section{Keywords}

Piezoelectric transducer, mounting, electromechanical impedance, damage, structural health monitoring

\section{Introduction}

In the modern world, there are many structural systems that people use daily such as civil infrastructure and the various major means of transport. Inevitably, these structures suffer aging, which can change their ideal operating conditions or prejudice their safe use. A structural health monitoring (SHM) system is capable of detecting structural damage and thus improving the safety of structures and reducing their corresponding maintenance costs (Bakht and Mufti, 2015; Farrar and Worden, 2013).

A complete SHM system consists of a sensor network; a data acquisition (DAQ), storage, and processing platform; and a method of detecting damage or changes in structural behavior. It is desirable for the structural damage to be detected using a nondestructive method (Giurgiutiu, 2013; Waugh, 2016), known as nondestructive evaluation (NDE) or nondestructive testing (NDT). Numerous methods for the detection of structural damage, such as acoustic emission
(AE; Ohtsu et al., 2016), Lamb waves (Kim and Chattopadhyay, 2015), eddy currents (Arjun et al., 2015), and methods based on fiber optic sensors (Zhao et al., 2015), have been developed. Among the wide range of available methods, the electromechanical impedance (EMI) technique stands out for its use of low-cost, small, and lightweight piezoelectric transducers operating simultaneously as sensors and actuators (Albakri and Tarazaga, 2017; Na and Park, 2017).

The principle of the EMI technique is based on the piezoelectric effect (Meitzler et al., 1988). Using this effect and by attaching a piezoelectric transducer onto

Universidade Estadual Paulista (UNESP), Faculdade de Engenharia, Bauru, Brasil.

\section{Corresponding author:}

Fabricio Guimarães Baptista, Unesp-FEB, Departamento de Engenharia Elétrica, Av. Eng. Luiz Edmundo C. Coube I4-0I, I7033-360, Bauru-SP, Brasil.

Email: fabriciogb@feb.unesp.br 
the structure to be monitored, a relationship between the electrical properties of the transducer and the mechanical properties of the structure is established. Thus, the integrity of the structure can be evaluated from the measurement and analysis of the electrical impedance of the transducer. Typically, the transducers used in the EMI method are made of thin lead zirconate titanate (PZT) ceramics coated on both sides with metal films serving as electrodes. However, conventional PZT ceramics are rigid and brittle, making their application in structures with uneven surface unfeasible. To overcome this drawback, more recently, macrofiber composite (MFC) transducers were proposed as an alternative (Brunner et al., 2009; Shahab and Erturk, 2016). These devices are low-profile, flexible, and durable, making their use in many types of structures feasible.

The transducers play a fundamental role in achieving the efficient and reliable detection of structural damage. In this regard, various studies have reported the development and characterization (Cao et al., 2015), appropriate sizing (Baptista et al., 2011; Baptista and Vieira Filho, 2010b), and correct diagnosis and validation (Overly et al., 2009) of transducers for SHM applications. Equally important is the way in which the transducers are installed in the structure. Therefore, the objective of this study is to analyze the effects of the transducer mounting on the detection of damage in SHM applications based on the EMI method.

Usually, the basic procedure for installing a transducer in a structure is using a high-stiffness adhesive such as epoxy or cyanoacrylate glue. This basic method of mounting has been widely used and investigated in the literature such as in the development of electromechanical models (Annamdas and Annamdas, 2009; Liang et al., 1994) and analysis of the effects of the adhesive layer (Moharana and Bhalla, 2015). The direct installation of the transducer in the structure using adhesive, although simple, has the disadvantage of making the transducer difficult to remove, possibly damaging the transducer or the structure. Furthermore, the direct mounting of the transducer is not always possible in structures under adverse operating conditions. To avoid such problems, many researchers have proposed alternative methods for mounting the transducers.

$\mathrm{Na}$ et al. (2012), for example, proposed mounting the transducer via a magnetic coupling. The authors fixed the PZT ceramic in a small magnet, which is attached to the structure by magnetic attraction using a second magnet. The simple solution proposed by the authors enables the reuse of the transducer and its easy repositioning in the structure if necessary.

The use of a steel wire for coupling the transducer to the structure was proposed by Na and Lee (2013). The authors attached a PZT patch at the end of a steel wire in the longitudinal direction, and they attached the other end to the surface of the monitored structure.
More recently, Naskar and Bhalla (2016) presented a more detailed experimental and numerical investigation of this mounting method, wherein the steel wire was replaced by metal foils with different cross sections. The authors reported a reduction in the sensitivity for damage detection compared to conventional transducer mountings. However, this method has the advantage of allowing for the monitoring of inaccessible structures, with irregular surfaces or under adverse operating conditions (e.g. high temperatures) for which the conventional method of transducer mounting would not be possible. This mounting method is commonly called metal-wire-based electromechanical impedance (MWBEMI).

Therefore, in this article, we present a comparative evaluation of three transducer mounting methods: conventional direct mounting, magnetic mounting and MWBEMI. Tests were performed on aluminum beams, and the performances of the three mounting methods were compared using the pencil-lead-break (PLB) test, the electrical impedance signatures, and the basic damage index.

In this study, two damage detection methods were evaluated. In addition to the conventional EMI method, where a single transducer operates as both a sensor and an actuator, we also tested a variation in this method called the transfer frequency response function (FRF) method, as reported by Martowicz et al. (2016). In the transfer FRF method, two transducers are used, one operating as the actuator and the other operating as the sensor. Damage detection is based on the analysis of the FRF signatures obtained from the relation between the excitation and response signals from both transducers in the frequency domain. The background of the conventional EMI and transfer FRF methods is presented in the next section.

\section{EMI method}

As mentioned above, two variants of damage detection based on the impedance method were considered in this study: the conventional EMI and the transfer FRF.

\section{Conventional EMI method}

The principle of damage detection based on the EMI method consists of attaching a piezoelectric transducer to the structure to be monitored. Figure 1 shows the basic configuration used in the conventional EMI method, where a PZT patch, commonly used as a transducer, is attached to a structure.

According to Figure 1, a measurement system simultaneously excites the transducer while providing its electrical impedance $\left(Z_{E}(\omega)\right)$. Therefore, in the EMI method, the transducer operates simultaneously as the sensor (direct piezoelectric effect) and the actuator (reverse piezoelectric effect). As is well known, the 


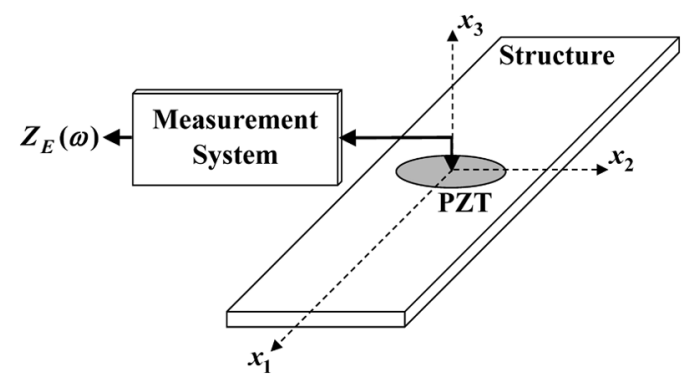

Figure I. Basic configuration used in the conventional EMI method.

deformation of the piezoelectric material occurs in all directions. However, because the thickness of the PZT patch is usually very small (a fraction of a millimeter), the deformation in the direction of its thickness $\left(x_{3}\right.$ axis in Figure 1) may be disregarded at sufficiently low frequency. It is important to note that for measured impedances at high frequencies (in the $\mathrm{MHz}$ range), also known as thickness-mode EMI, deformations along the thickness of the material can be detected (Kamas and Tekkalmaz, 2016).

Furthermore, based on a one-dimensional assumption and considering a narrow structure, only a deformation in the longitudinal direction ( $x_{1}$ axis in Figure 1$)$ can be considered, disregarding the deformation along the $x_{2}$ axis.

Based on these assumptions, the direct piezoelectric effect (sensor) and reverse piezoelectric effect (actuator) in the transducer are given by equations (1) and (2), respectively

$$
\begin{gathered}
D_{3}=d_{31} T_{1}+\varepsilon_{33}^{T} E_{3} \\
S_{1}=s_{11}^{E} T_{1}+d_{31} E_{3}
\end{gathered}
$$

where $D_{3}$ and $E_{3}$ are the electrical displacement and the electric field, respectively; $T_{1}$ and $S_{1}$ are the mechanical stress and strain, respectively; and $d_{31}, \varepsilon_{33}^{T}$, and $s_{11}^{E}$ are the piezoelectric, dielectric, and elastic compliance constants of the piezoelectric material, respectively, where the superscripts $T$ and $E$ indicate constant stress and constant electric field, respectively, and the subscripts 1 and 3 represent the axes of the natural coordinate system of the piezoelectric material under the onedimensional assumption.

From equations (1) and (2), a one-dimensional electromechanical model (Liang et al., 1994) may be derived to relate the electrical properties of the transducer with the mechanical properties of the structure. Thus, the electrical impedance of a generic transducer calculated using a simplified version of this model is given by

$$
Z_{E}(\omega)=\frac{1}{j \omega C}\left(1-\frac{d_{31}^{2}}{s_{11}^{E} \varepsilon_{33}^{T}} \frac{Z_{S}(\omega)}{Z_{S}(\omega)+Z_{P}(\omega)}\right)^{-1}
$$

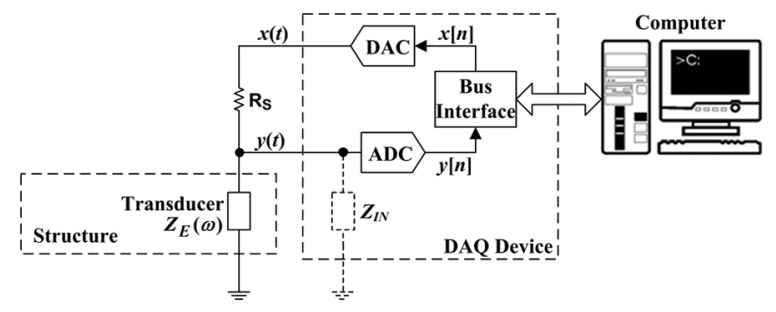

Figure 2. Measurement system used in the conventional EMI method.

where $Z_{E}(\omega)$ is the electrical impedance of the transducer at angular frequency $\omega$, which should vary within an appropriate range provided by the measuring system; $Z_{P}(\omega)$ is the mechanical impedance of the transducer; $Z_{S}(\omega)$ is the mechanical impedance of the monitored structure; $C$ is the static capacitance of the transducer; and $j$ is the unit imaginary number.

Structural damage, such as cracks, corrosion, and the loosening of connections, causes variations in the mechanical impedance of the structure. According to equation (3), any variation in the mechanical impedance of the structure $\left(Z_{S}(\omega)\right)$ causes a corresponding change in the electrical impedance of the transducer $\left(Z_{E}(\omega)\right)$, known as EMI. Therefore, the structural health can be monitored by measuring and analyzing the electrical impedance of the transducer, which is easy to perform.

The measurement of the electrical impedance of the transducer may be performed using a commercial impedance analyzer. However, many researchers have proposed alternative measurement systems based on time-domain (Vieira Filho et al., 2011) or frequencydomain (Cortez et al., 2013; Kaur et al., 2016; Maruo et al., 2015) analysis. In this study, the electrical impedance was measured using the alternative system proposed by Baptista and Vieira Filho (2009), which is based on a multifunctional DAQ device controlled by a computer running appropriate software and drivers for the utilized DAQ model. The basic configuration of this system is shown in Figure 2.

As seen in Figure 2, the DAQ device must have at least one analog output for providing the excitation signal $(x(t))$ through the digital-to-analog converter (DAC) and one analog input for the acquisition of the response signal $(y(t))$ from the transducer through the analog-todigital converter (ADC). The signals $x[n]$ and $y[n]$ are the digital forms of the excitation and response signals, respectively, which are processed in the computer. The connection between the DAQ device and the computer is achieved via a standard bus such as universal serial bus (USB), peripheral component interconnect (PCI), and peripheral component interconnect express (PCIe). The transducer is connected through a resistor $R_{S}$.

The impedance of the analog input $\left(Z_{I N}\right)$ may be ignored if it is sufficiently high compared with the 
impedance of the transducer. In addition, the wiring resistance can also be disregarded for short connections. Based on these considerations, the electrical impedance of the transducer can be calculated as

$$
Z_{E}(\omega)=R_{S} \frac{H(\omega)}{1-H(\omega)}
$$

where $H(\omega)$ is the FRF, taking the excitation signal $(x[n])$ as input and the response signal $(y[n])$ as output, which is calculated as follows

$$
H(\omega)=\frac{S_{x y}(\omega)}{S_{x x}(\omega)}
$$

where $S_{x y}(\omega)$ is the crossed power spectrum between the excitation signal $(x[n])$ and the response signal $(y[n])$ and $S_{x x}(\omega)$ is the auto power spectrum of the excitation signal, which are given by

$$
\begin{aligned}
& S_{x y}(\omega)=\frac{X(\omega) Y^{*}(\omega)}{N} \\
& S_{x x}(\omega)=\frac{X(\omega) X^{*}(\omega)}{N}
\end{aligned}
$$

where $X(\omega)$ and $Y(\omega)$ are the discrete Fourier transform (DFT) of the excitation signal $(x[n])$ and the response signal $(y[n])$, respectively, computed using the fast Fourier transform (FFT) algorithm; $X^{*}(\omega)$ and $Y^{*}(\omega)$ denote the complex conjugates; and $N$ is the length of the signals.

It is important to note that in this measuring method, the amplitude voltage of the transducer $(y(t))$ is not constant; rather, it decreases with increasing frequency due to the capacitive reactance of the transducer $(1 / j \omega C)$, as shown in equation (3). From the electrical point of view, this does not affect the measured value of the impedance because the FRF $(H(\omega))$ does not depend on the signal amplitude, ensuring accurate measurements (Baptista and Vieira Filho, 2009). However, in the EMI technique, the impedance signatures consist of resonance peaks related to the monitored structure that can be affected by the signal amplitude, thereby altering the sensitivity to damage, especially in large structures.

Therefore, special attention is required in choosing the resistor $R_{S}$ in Figure 2 and the frequency band, particularly in the impedance measurements for detecting damage in large structures or in research that proposes to investigate the effects of the amplitude of the excitation signal. In these cases, the amplitude of the excitation signal must be as constant as possible, which can be achieved using a small resistor and performing the impedance measurement in narrow frequency bands.

Typically, the FRF is obtained by taking an average of several measurements. The excitation signal must be dynamic, such as a chirp signal, with the same frequency range desired for the electrical impedance signatures. The transducer is usually bonded to the host structure using an adhesive such as epoxy or cyanoacrylate glue. In this study, the impedance signatures were obtained from measurements of specimens to which the piezoelectric transducers were mounted using the three above-described mounting methods. In addition, the mounting methods were also analyzed for damage detection based on the transfer FRF method. In this method, two transducers are used, one operating as the actuator and the other as the sensor, as shown in the following section.

\section{Transfer FRF method}

In contrast to the conventional EMI method, which is known for using a single transducer, the transfer FRF method uses at least two transducers, each operating separately as an actuator and a sensor. Therefore, the input and output signals of the FRF are obtained at different points of the structure.

This method is particularly useful for detecting damage located between the two transducers. For example, Martowicz et al. (2016) used this method to monitor a bolted pipeline connection. The authors bonded pairs of PZT patches in steel washers to obtain the transfer FRFs to detect and quantify the loosening of the bolts. The results were compared with those obtained with the conventional EMI method, also called point FRF. The basic setup that we used to obtain the transfer FRF signatures is shown in Figure 3.

The measurement system is similar to that used in the conventional EMI method, with the difference being that the excitation signal $(x(t))$ is applied to the actuator transducer through the limiting resistor $\left(R_{S}\right)$ and that the response signal $(y(t))$ is obtained from the sensor transducer. The transfer FRF $H(\omega)$ is obtained from the corresponding digital signals $x[n]$ and $y[n]$ by computing the crossed power spectrum $\left(S_{x y}(\omega)\right)$ and the auto power spectrum $\left(S_{x x}(\omega)\right)$, as shown in equations (5) to (7). The excitation and response signals are generated and sampled simultaneously and synchronously.

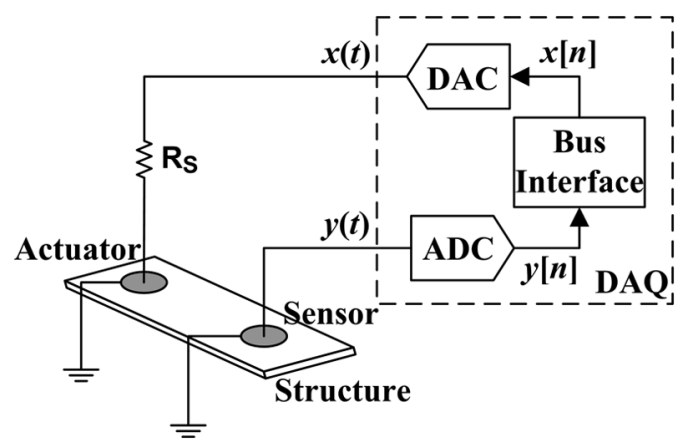

Figure 3. Basic setup used in the transfer FRF method. 
As mentioned above, the FRF $H(\omega)$ is obtained from an average of multiple measurements to obtain a good repetition among the signatures and minimize any noise effects. Structural damage, especially along the path between the two transducers, causes variations in the FRF signatures, which can be analyzed as conventional impedance signatures for monitoring the integrity of the structure.

In this study, the three mounting methods were tested using both the actuator transducer and the sensor transducer to analyze and compare the effects on the sensitivity for structural damage detection. The assessment of the sensitivity for damage detection was performed using the PLB test and damage indices, as described in the following section.

\section{Sensitivity assessment}

The evaluation of the transducer mounting methods in terms of effectiveness in the detection of structural damage was conducted using the PLB test, by comparing the electrical impedance and FRF signatures, and using damage indices.

\section{PLB test}

The PLB test is a well-known procedure in AE applications for the generation of an acoustic signal. The procedure consists of breaking a pencil lead against the structure or rod on which the transducer is installed. In this study, the pencil lead was broken against the monitored structures, which are aluminum specimens.

Upon breaking the pencil lead, an impulsive stress is released, and a wide-band signal is consequently generated, which can be used to evaluate the AE sensor response. When the pencil lead is broken, the voltage signal from the sensor is sampled. The analysis is usually performed in the frequency domain by computing the power spectral density (PSD). Therefore, the PLB test is an economical and simple procedure for obtaining an acoustic source and has been adopted as a standard (ASTM, 2010).

Although very common in AE applications, the PLB test has also been used to assess the sensitivity of piezoelectric transducers for damage detection based on the EMI method (De Almeida et al., 2015). In this study, we used the PLB test to assess the response of the piezoelectric transducers installed in the monitored structure using different mounting methods. The pencil lead was broken in the monitored structure in the same position and under the same conditions for all mounting methods so that the differences observed between the signals obtained from the transducers were primarily due to the mounting method. The analysis was performed by calculating and comparing the PSDs of the three mounting methods.

\section{Damage indices}

A basic way to detect and quantify structural damage in the EMI method is by comparing two electrical impedance signatures, one of which is obtained when the structure is in a state considered healthy, known as the baseline. This comparison is typically accomplished using damage indices. One of the most widely used indices is the root-mean-square deviation (RMSD), which is based on the Euclidean distance between the two signatures. The RMSD index is calculated as (Farrar and Worden, 2013)

$$
\operatorname{RMSD}=\sum_{\omega=\omega_{I}}^{\omega_{F}} \sqrt{\frac{\left[\operatorname{Re}\left(Z_{2}(\omega)\right)-\operatorname{Re}\left(Z_{1}(\omega)\right)\right]^{2}}{\operatorname{Re}\left(Z_{1}(\omega)\right)^{2}}}
$$

where $\operatorname{Re}\left(Z_{1}(\omega)\right)$ is the real part of the baseline signature, $\operatorname{Re}\left(Z_{2}(\omega)\right)$ is the real part of the impedance signature after possible damage, and RMSD is the index calculated in the frequency range with initial frequency $\omega_{I}$ and final frequency $\omega_{F}$.

The RMSD index may also be calculated using the magnitude or the imaginary part of the impedance; however, the real part is normally used because it is more sensitive to damage and less sensitive to temperature (Baptista et al., 2014; Park et al., 2003). Despite this, for the transfer FRF method, the index was calculated using the magnitude of the FRF, $|H(\omega)|$, because it is more representative in this method.

Another widely used index is the correlation coefficient deviation metric (CCDM), which is based on the correlation coefficient between the two impedance signatures and is calculated as

$$
\mathrm{CCDM}=1-C_{C}
$$

where $C_{C}$ is the correlation coefficient, given by

$$
C_{C}=\frac{\operatorname{cov}\left[\operatorname{Re}\left(Z_{1}(\omega)\right), \operatorname{Re}\left(Z_{2}(\omega)\right)\right]}{\sigma_{1} \sigma_{2}}
$$

in which "cov" is the covariance of the real parts of the two impedance signatures as previously defined, calculated in the same frequency range as the RMSD index in equation (8), and $\sigma_{1}$ and $\sigma_{2}$ are the corresponding standard deviations of each signature.

The RMSD and CCDM indices were calculated for all mounting methods under the same experimental conditions and compared to evaluate the influence of the transducer mounting on the detection of structural damage. The experimental setup is shown in the next section.

\section{Experimental setup}

To analyze the effects of the transducer mounting on the detection of structural damage, tests were performed on $1100 \times 76 \times 3 \mathrm{~mm}^{3}$ aluminum beams. 


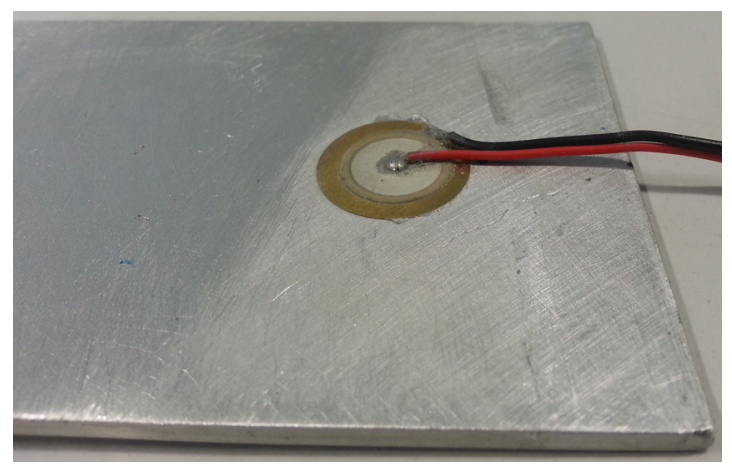

Figure 4. Conventional mounting method.

The transducers used in this study are piezoelectric diaphragms, commonly known as "buzzers," consisting of a 0.20 -mm-thick, $20 \mathrm{~mm}$ in diameter circular brass plate and a $0.22-\mathrm{mm}$-thick, $14 \mathrm{~mm}$ in diameter piezoelectric ceramic (active element). The main application of these devices is the generation of various types of sounds, such as alarms and beeps; however, many recent scientific studies have reported the feasibility of using piezoelectric diaphragms for structural damage detection based on the EMI and other methods (Freitas and Baptista, 2016; Tinoco and Marulanda, 2015).

For the conventional direct mounting method, the transducer was bonded directly to the structure using cyanoacrylate glue at a distance of $30 \mathrm{~mm}$ from the center of the transducer and the end of the beam. The conventional transducer mounting is shown in Figure 4.

In the mounting method using magnets, the transducer was bonded to a 3-mm-thick, $20 \mathrm{~mm}$ in diameter circular neodymium magnet using cyanoacrylate glue. The coupling to the structure was achieved by placing another magnet of the same size on the bottom side in the structure at the position equivalent to that of the conventional method. This procedure is shown in Figure 5.

Finally, to assess the MWBEMI method in which the coupling between the transducer and the structure to be monitored is accomplished using a wire or metal foil, two aluminum foils with a length of $300 \mathrm{~mm}$, a width of $30 \mathrm{~mm}$, and different thicknesses of 0.5 and $1.0 \mathrm{~mm}$ were tested. A transducer was attached at a distance of $30 \mathrm{~mm}$ from one end of each foil, and the other end was attached to the structure at a position equivalent to those of the other methods. In this method, cyanoacrylate glue was also used to fasten the transducer to the foils and the foils to the structure. The MWBEMI mounting is shown in Figure 6.

The measurement of the electrical impedance was conducted with the measuring system based on a DAQ device (Baptista and Vieira Filho, 2009) according to the simplified diagram shown in Figure 2 using the multifunctional DAQ NI-USB-6361. The transducers were excited through a $2.2-\mathrm{k} \Omega$ resistor $\left(R_{S}\right.$ in Figure 2$)$ and a

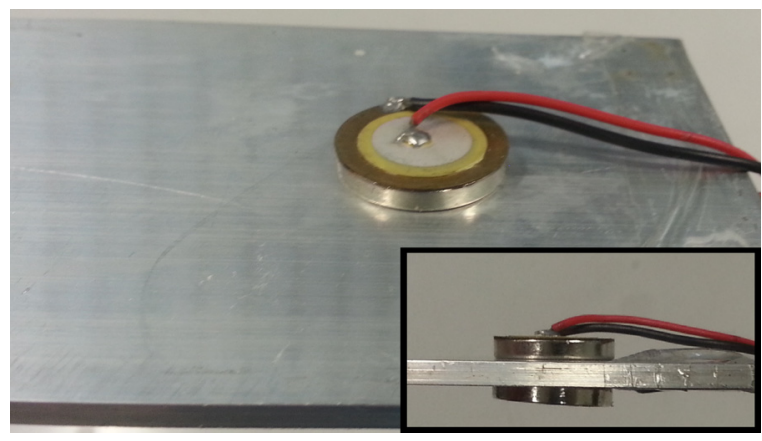

Figure 5. Magnetic mounting method.

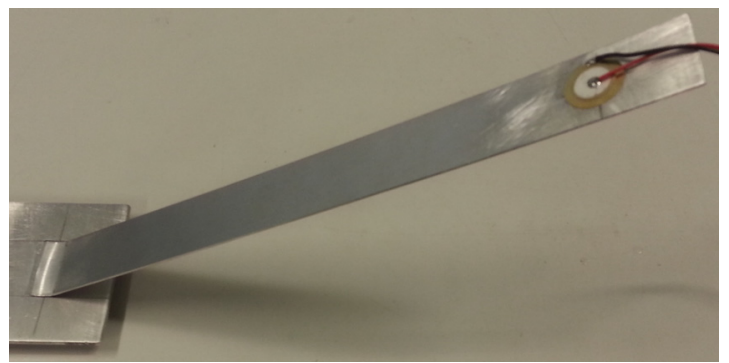

Figure 6. MWBEMI mounting method.

chirp signal with an amplitude of $1 \mathrm{~V}$ and frequency range of $0-500 \mathrm{kHz}$. The $2.2-\mathrm{k} \Omega$ resistor is suitable for the experimental conditions of this study. As mentioned above, although the signal amplitude of the transducer varies with the frequency, the influence on the sensitivity to damage is negligible because the monitored structure is small. Moreover, the same signal level was used in all transducer mounting methods, ensuring a proper comparison.

The response signals from the transducers were sampled at a sampling rate of $2 \mathrm{MS} / \mathrm{s}$. The electrical impedance signatures were obtained in the frequency range of $0-500 \mathrm{kHz}$, therein taking the average of 5 measurements, which ensures good accuracy and precision.

Structural damage was induced by fixing a metal mass $(14 \mathrm{~mm} \times 8 \mathrm{~mm}$ steel screw nut) in the structure using cyanoacrylate glue at distances of 100 and $1000 \mathrm{~mm}$ from the transducers bonded to the substrate structure using each mounting method. This procedure changes the mechanical impedance of the structure similarly to structural damage but provides the advantage of not permanently damaging the structure.

For the transfer FRF method, the system was modified as shown in Figure 3 by installing a second transducer using the corresponding mounting method at the other end of the aluminum beam, therein keeping the DAQ device settings unchanged. In this method, one of the transducers operated as an actuator, while the other operated as a sensor. The FRF signatures $(H(\omega))$ were obtained in the range of $0-500 \mathrm{kHz}$ as the average of 


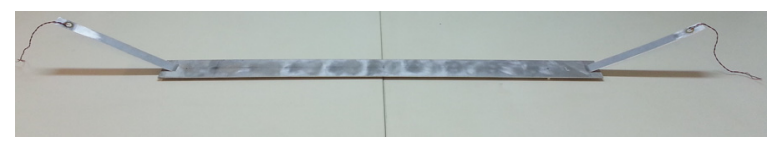

Figure 7. Transfer FRF method using the MWBEMI mounting.

five measurements. Structural damage was induced by fixing the same metal mass in the middle of the structure between the two transducers. As an example, Figure 7 shows the experimental arrangement of the transfer FRF method using the MWBEMI mounting, where aluminum foils achieve the coupling between the transducers (sensor and actuator) and the structure.

In the PLB test, we used a mechanical pencil provided with lead with a length of $3 \mathrm{~mm}$ and a diameter of $0.5 \mathrm{~mm}$. The lead break was performed manually, with an angle between the structure and the lead of approximately $45^{\circ}$ and at a distance of $100 \mathrm{~mm}$ from the transducer or equivalent mounting method. When the lead was broken, the signal voltage from the transducers with the different mounting methods was sampled at a sampling rate of $2 \mathrm{MS} / \mathrm{s}$. Because the signal is impulsive, a passive resistor/capacitor (RC) antialiasing filter with a $677 \mathrm{kHz}$ cutoff frequency was used. The analysis was performed in the frequency domain by computing the PSD similarly as in the previous study (De Almeida et al., 2015) using the Welch method with a 256-point Hanning window with a $50 \%$ overlap and by taking an average of 10 measurements.

In all the tests, the measurements were conducted at room temperature of approximately $25^{\circ} \mathrm{C}$ stabilized using an air conditioner, and the specimens were supported on a desk using rubber blocks. The results are presented and discussed in the following section.

\section{Results and discussion}

\section{PLB test}

As mentioned above, the PLB test is a well-known method for assessing the frequency response of transducers in AE applications. In this study, this test was

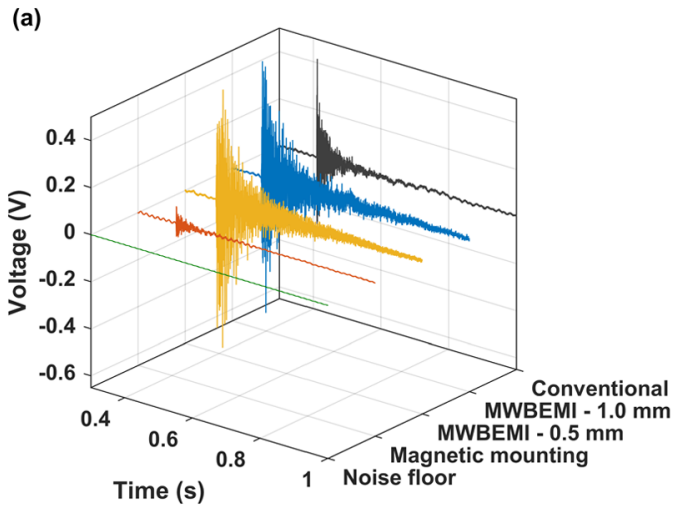

used to assess the sensitivity of the transducers with three different mounting methods and the effects of the mounting methods on structural damage detection. Because the test was performed under the same conditions in all cases, the differences observed in the results may be attributed primarily to the mounting method.

The voltage signals in the time domain obtained for the different mounting methods are shown in Figure 8(a), and the corresponding PSDs in the frequency domain are shown in Figure 8(b) compared to the noise floor. The noise floor was obtained by taking an average of 10 measurements without any activity, that is, without performing the PLB test.

In the time domain, the signals from the transducers when using the conventional and MWBEMI mounting are similar in terms of the voltage level. In contrast, the signal obtained from the transducer mounted with magnets exhibited a significantly lower voltage. The same behavior is observed in the PSDs in the frequency domain. In all mounting methods, the PSDs are higher for low frequencies and decrease with increasing frequency. In general, for frequencies up to approximately $100 \mathrm{kHz}$, the magnetic mounting provided the lowest PSD, and the conventional direct mounting showed a slightly higher PSD compared to both MWBEMI mountings, except at frequencies below approximately $10 \mathrm{kHz}$. For frequencies above $100 \mathrm{kHz}$, the PSDs of all mounting methods are low and similar to each other and have an average value approximately $20 \mathrm{~dB}$ higher than the noise floor.

Therefore, in general, a higher sensitivity to structural damage using the conventional mounting and a lower sensitivity using the magnetic mounting are expected. The analysis of the effects of the transducer mounting on the detection of structural damage based on the EMI method is presented and discussed in the following section.

\section{Conventional EMI method}

To analyze the effects of the mounting methods on the impedance signatures and, consequently, on the

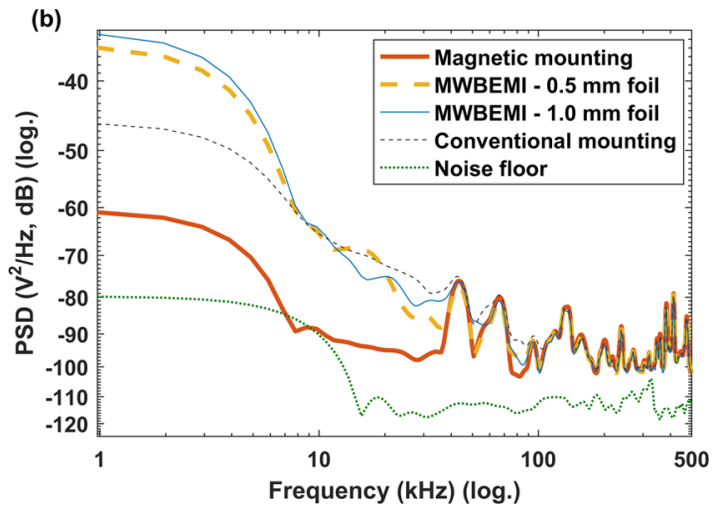

Figure 8. (a) Voltage signals and (b) PSD obtained for each mounting method using the PLB test and compared to the noise floor. 


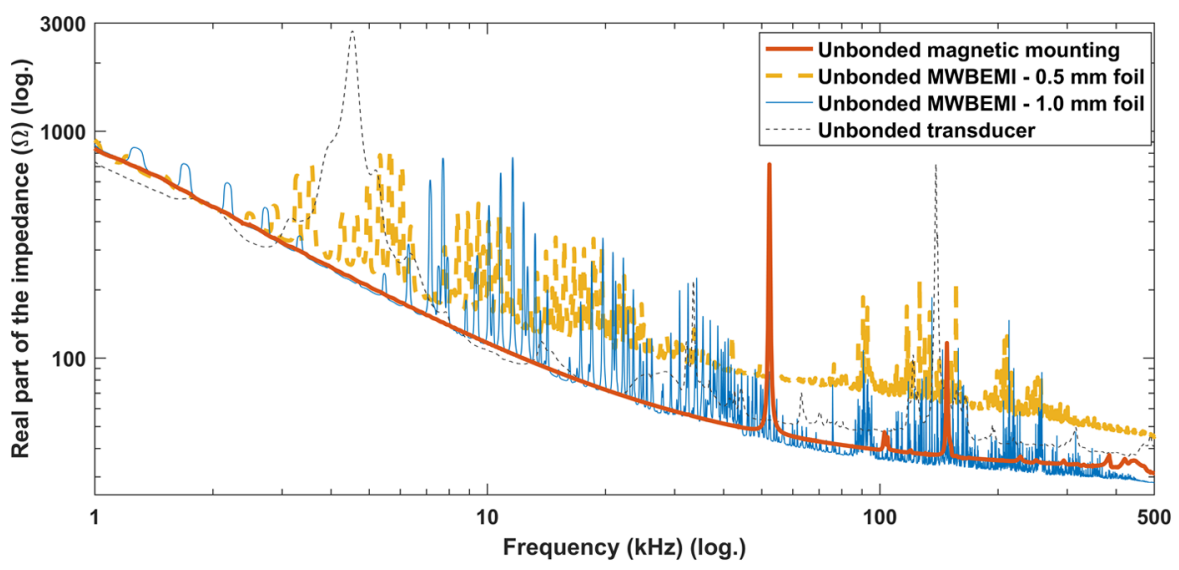

Figure 9. Real part of the impedance signatures obtained under the unbonded condition.

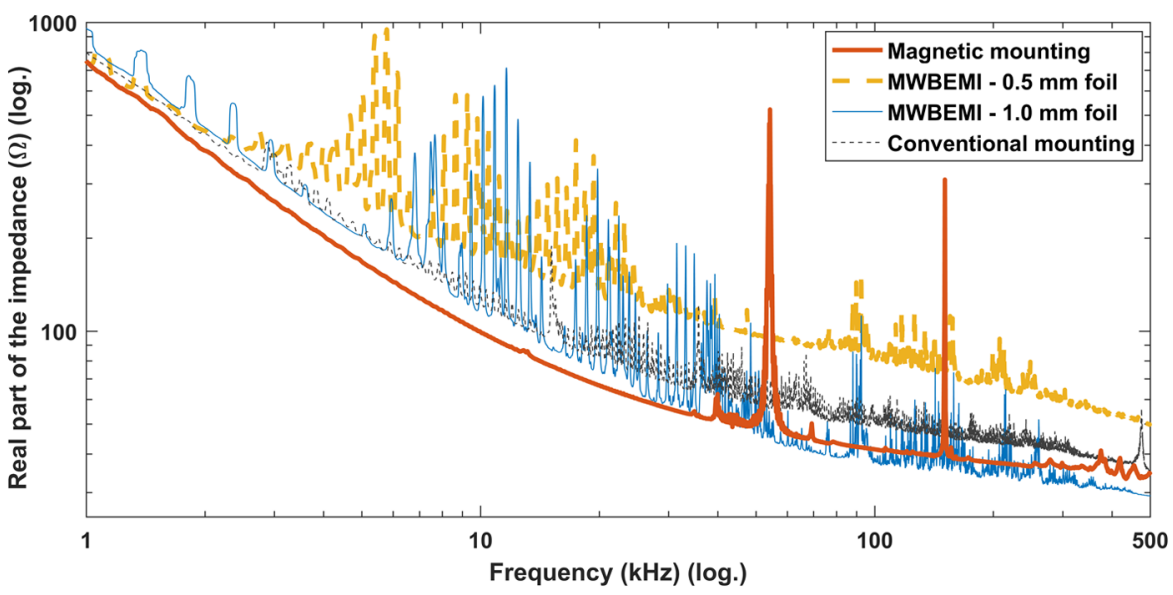

Figure 10. Real part of the impedance signature obtained for each mounting method bonded to the monitored structure.

detection of structural damage, the electrical impedance signatures were initially measured with the free piezoelectric transducer and the individual mounting solutions unbonded from the monitored structures (aluminum beams). The real parts of the impedance signatures, which are most widely used in the literature, are shown in Figure 9.

According to Figure 9, the impedance signatures present resonance peaks related mainly to the transducer holder and the structure of each mounting method. The impedance signature of the transducer exhibits few prominent resonance peaks related to its circular brass plate. Similarly, the impedance signature of the magnetic mounting exhibits only two significant peaks, at frequencies of approximately 54 and $150 \mathrm{kHz}$, related to the utilized magnet. In contrast, the impedance signatures obtained for the MWBEMI method have a large number of resonance peaks, related to the thin aluminum foils.

Then, the impedance signatures were measured with the transducer and the mounting methods bonded to the monitored structures (aluminum beams). The results are shown in Figure 10.
Comparing the impedance signatures in Figures 9 and 10 , we observe that the signature obtained for the transducer is significantly different when it is directly bonded to the structure (conventional mounting), increasing the number of resonance peaks, mainly at frequencies above $10 \mathrm{kHz}$. This result indicates an effective coupling between the transducer and the monitored structure since the impedance signature begins to show resonance peaks related primarily to the structure. In contrast, significant changes are not observed in the signatures under the other mounting methods when they are bonded to the structure, indicating a less effective coupling between the transducer and the monitored structure compared to the conventional mounting.

Therefore, according to the experimental results, the transducer mounting changes the impedance signatures significantly. The resonance peaks become predominant due to the mounting structure, which is the neodymium magnet or aluminum foil. In addition to changes in the impedance signatures caused by the mounting method, it is important to analyze the impact of each method on the sensitivity to structural damage. A qualitative assessment of the sensitivity of each mounting 

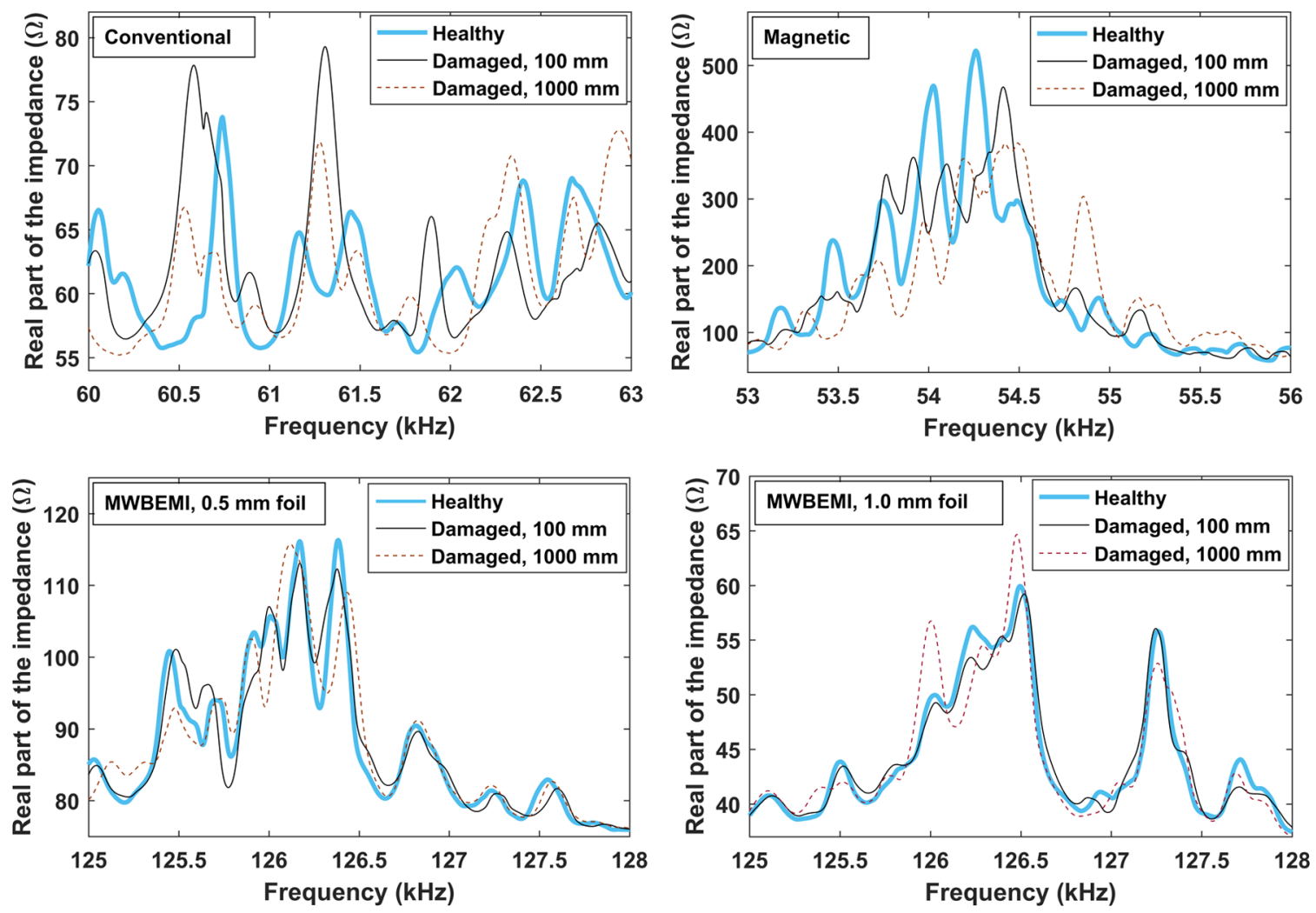

Figure I I. Impedance signatures obtained for the structure under the healthy and damaged conditions.

method for detecting structural damage can be performed by comparing the changes in the impedance signatures due to the occurrence of damage. Figure 11 shows a comparison between the impedance signatures obtained for the structure in a healthy state and after the damage induction at distances of 100 and $1000 \mathrm{~mm}$ from each mounting method.

Only narrow frequency bands more sensitive to damage under each mounting method are shown in Figure 11 to ensure a proper comparison. According to the results, all mounting methods are able to detect structural damage because variations are observed in the impedance signatures due to the occurrence of damage. However, the conventional mounting provides more prominent variations both in amplitude and in the shape of the signatures. Moreover, the alternative mounting methods cause the damage detection to be more selective at some frequency bands. For example, the magnetic mounting is sensitive to damage at narrow frequency bands around the resonance peaks at frequencies of approximately 54 and $150 \mathrm{kHz}$, as shown previously in Figures 9 and 10.

The effects of the transducer mounting on the damage detection can be more quantitatively and effectively evaluated by computing the RMSD and CCDM damage indices given by equations (8) and (9), respectively. As is well known, the selection of the most sensitive frequency range for damage detection is a critical process
(Baptista and Vieira Filho, 2010a) that depends on the structure, the transducer, and the mounting method. To assess the effects of the transducer mounting across the frequency range, the indices were calculated in subbands of $25 \mathrm{kHz}$ across the range of $0-500 \mathrm{kHz}$, in which the impedance signatures were measured. Figure 12 shows the RMSD indices obtained for damage (steel nut) at distances of (a) $100 \mathrm{~mm}$ and (b) $1000 \mathrm{~mm}$ from the transducer or equivalent mounting.

According to Figure 12, in the conventional and MWBEMI mounting methods, there is a predominance of high RMSD indices at low frequencies. This result is in agreement with the PSDs shown in Figure 8, where the higher values are mainly concentrated at low frequencies, and the impedance signatures shown in Figures 9 and 10, where there is a large number of resonance peaks at low frequencies. Overall, the magnetic mounting provided low RMSD indices compared to other mountings, except in the sub-bands of 50-75 and $150-175 \mathrm{kHz}$, where pronounced RMSD indices are observed. This result is also consistent with the PSD and impedance signatures observed in Figures 8 to 10 . Under the experimental conditions considered in this study, the distance between the damage and the transducer or the equivalent mounting did not cause significant changes in the RMSD indices. The results obtained with the CCDM index are shown in Figure 13. 

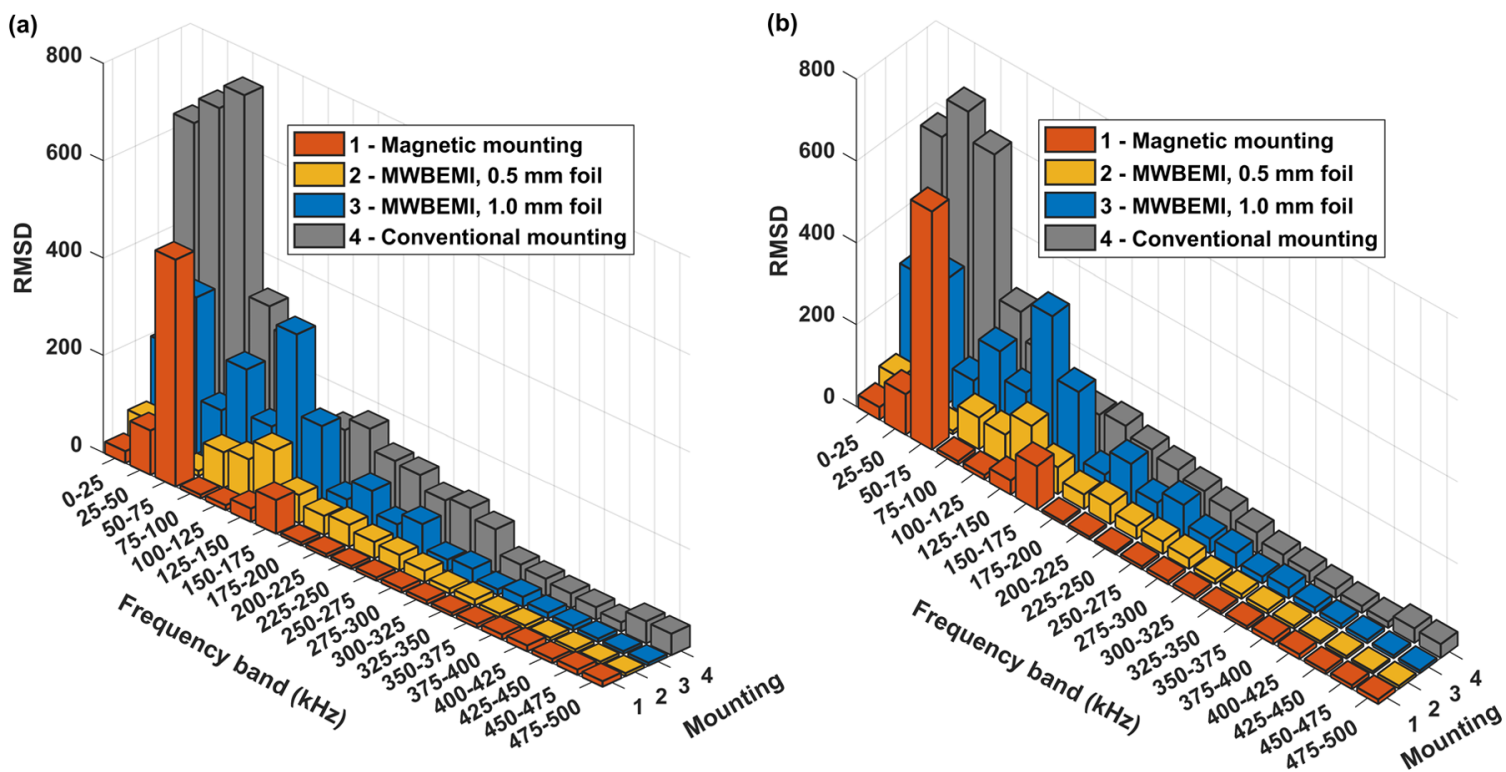

Figure 12. RMSD indices obtained for damage at distances of (a) $100 \mathrm{~mm}$ and (b) $1000 \mathrm{~mm}$ from the transducer or mounting.

(a)

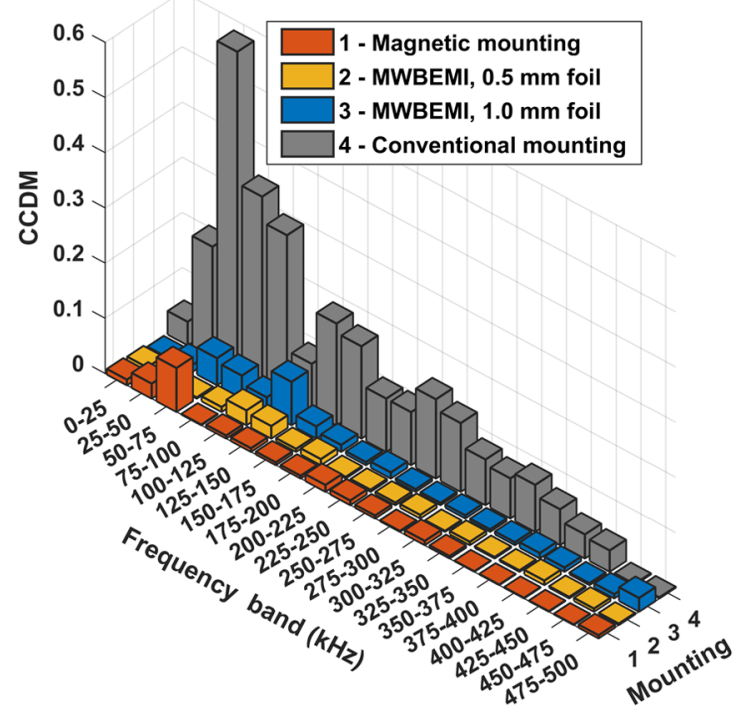

(b)

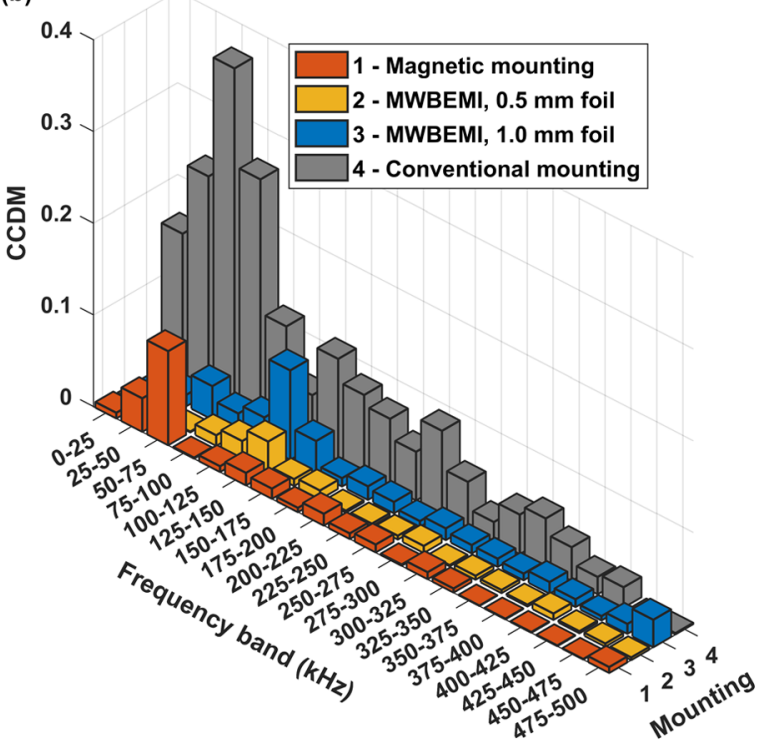

Figure 13. CCDM indices obtained for damage at distances of (a) $100 \mathrm{~mm}$ and (b) $1000 \mathrm{~mm}$ from the transducer or mounting.

The results obtained based on the CCDM index are similar to those obtained with the RMSD index, although the differences between the indices obtained with the conventional mounting and the other mountings were more pronounced. The distance between the structural damage and the transducer or equivalent mounting did not cause significant changes in the detection of damage; however, changes were observed in the amplitude of the indices.

Therefore, the results indicate that the conventional mounting is more sensitive for structural damage detection based on the EMI method. The MWBEMI mounting has an intermediate sensitivity that significantly depends on the thickness of the metal foil, as seen in the difference in the RMSD and CCDM indices obtained from the use of foil with thicknesses of 0.5 and $1.0 \mathrm{~mm}$. Finally, the magnetic mounting is less sensitive, although good sensitivity is observed in certain frequency bands. The results obtained for damage detection based on the transfer FRF are shown in the following section.

\section{Transfer FRF method}

The analysis of the effects of the transducer mounting on the damage detection based on the transfer FRF 

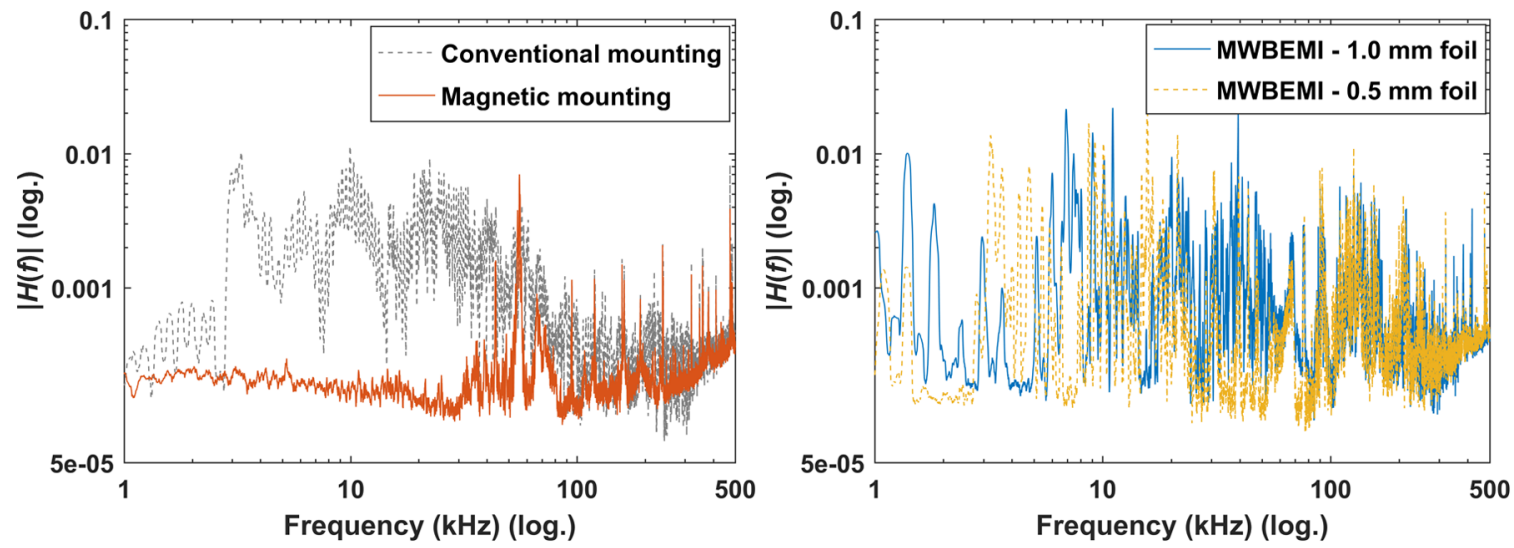

Figure 14. Magnitudes of the FRF signatures obtained for each mounting method.
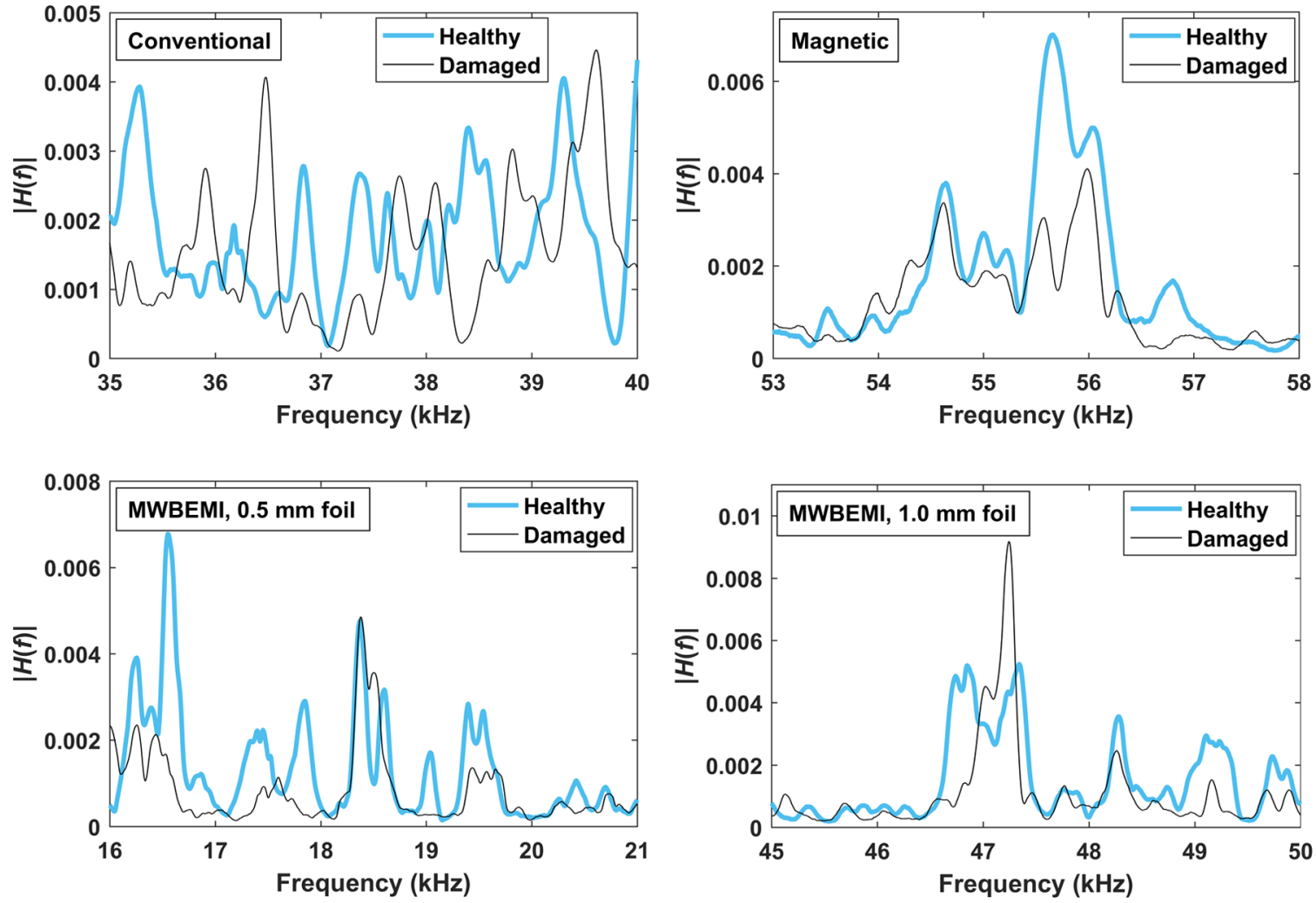

Figure 15. FRF signatures obtained for the structure under the healthy condition and under the damaged condition.

method was conducted similarly to the analysis performed for the conventional EMI method. Therefore, the sensitivity of each transducer mounting for damage detection was assessed based on damage indices calculated using the FRF signatures. The magnitudes of the FRF signatures obtained for the different transducer mountings are shown in Figure 14.

According to Figure 14, in general, the signature obtained when using the magnetic mounting presents low values compared to the other mountings, except around the frequency of $54 \mathrm{kHz}$, where the FRF is more pronounced, as also observed in the impedance signature.

A comparison between the FRF signatures obtained for the structure in the healthy state and for damage located in the center of the structure, as detailed previously, is shown in Figure 15. Only narrow frequency bands more sensitive to damage under each mounting method are shown for clarity.

According to Figure 15, the structural damage caused variations in the FRF signatures of all mounting methods, indicating the feasibility for detecting damage. 

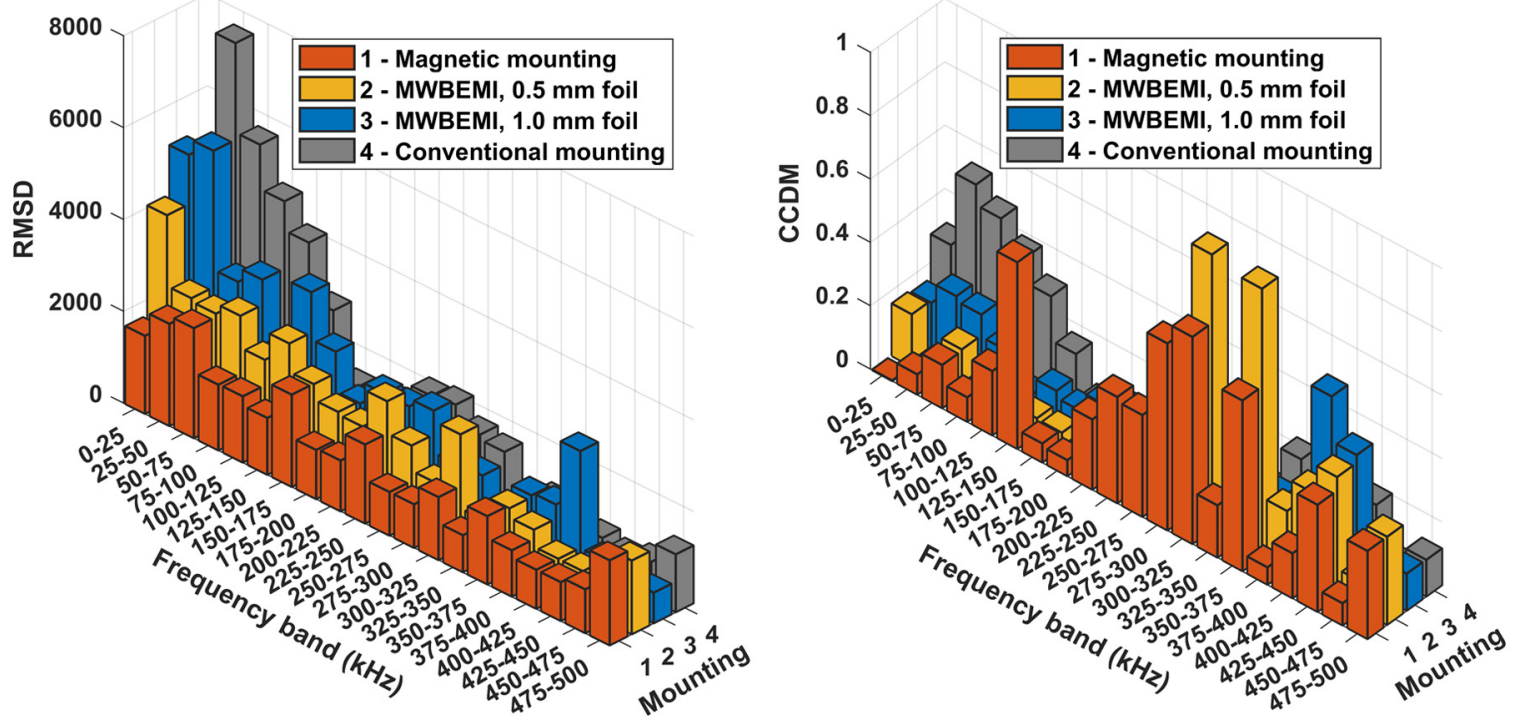

Figure 16. RMSD and CCDM indices obtained for the transfer FRF method.

Qualitatively, the conventional mounting provides slightly more prominent variations in the shape and amplitude between the healthy and damaged signatures. Similar to the results obtained with the conventional EMI method, the magnetic mounting provides less significant variation between the two FRF signatures.

To perform a quantitative analysis of the sensitivity of each mounting method to structural damage, the RMSD and CCDM indices were calculated in the subband of $25 \mathrm{kHz}$. The results are shown in Figure 16.

According to the results, the type of transducer mounting affects structural damage detection based on the transfer FRF method. In general, the RMSD indices are higher when using the conventional mounting method, indicating a higher sensitivity to damage for this type of mounting. In contrast, the magnetic mounting presented lower indices, indicating a low sensitivity to damage. The indices obtained using the MWBEMI mounting had intermediate levels, indicating that this type of mounting has an intermediate sensitivity compared to other types of mountings. Therefore, the effects of the transducer mounting on damage detection based on the transfer FRF method are similar to those using the conventional EMI method. However, as seen in Figure 16, the differences in the RMSD indices are smaller between the mounting types, indicating a less significant effect on the sensitivity compared to the conventional EMI method. Similarly, the differences in the CCDM indices are less prominent between the mounting methods and are without selective frequency subbands more sensitive to damage, as observed with the conventional EMI technique.

Furthermore, the RMSD and CCDM indices obtained for the transfer FRF method are significantly higher compared to the conventional EMI technique, especially at high frequencies. In the EMI technique, the transducer operates simultaneously as actuator and sensor, and the impedance signatures are directly related to its capacitive reactance $(1 / j \omega C)$, as shown in equation (3). Consequently, the variations in the impedance signatures are less significant, especially at high frequencies, where the capacitive reactance is low, as theoretically detailed in previous studies (Baptista et al., 2011; Baptista and Vieira Filho, 2010a). This explains the overall decrease in the RMSD and CCDM indices with increasing frequency, as shown in Figures 12 and 13, respectively. In contrast, the transfer FRF method uses two transducers, one of which is used as a passive sensor. Therefore, the FRF signatures are not directly related to the capacitive reactance of the transducer as in the impedance signatures, allowing for more significant variations due to damage, especially at high frequencies, as shown by the RMSD and CCDM indices in Figure 16.

The results for all mounting methods are summarized in Table 1, which highlights the highest PSD obtained in the PLB test and the highest RMSD and CCDM indices obtained using the conventional EMI and transfer FRF methods.

As noted in Table 1 and the graphs presented previously, the distance between the damage and the mounting methods has no significant influence on the damage indices. In some cases, the indices obtained for damage at a distance of $1000 \mathrm{~mm}$ were higher than those obtained for damage at a distance of $100 \mathrm{~mm}$. However, it is important to note that the structure used in this study is a small aluminum beam, and different results may be obtained with large structures. 
Table I. Maximum values obtained for each mounting method in all experimental tests.

\begin{tabular}{|c|c|c|c|c|c|c|c|}
\hline \multirow{3}{*}{$\begin{array}{l}\text { Transducer mounting } \\
\text { method }\end{array}$} & \multirow{3}{*}{$\begin{array}{l}\text { PLB test } \\
\text { PSD }(\mathrm{dB})\end{array}$} & \multicolumn{4}{|c|}{ Conventional EMI method } & \multicolumn{2}{|c|}{ Transfer FRF } \\
\hline & & \multicolumn{2}{|l|}{ RMSD } & \multicolumn{2}{|l|}{ CCDM } & \multirow[b]{2}{*}{ RMSD } & \multirow[b]{2}{*}{ CCDM } \\
\hline & & $\begin{array}{l}\text { Damage at } \\
100 \mathrm{~mm}\end{array}$ & $\begin{array}{l}\text { Damage at } \\
1000 \mathrm{~mm}\end{array}$ & $\begin{array}{l}\text { Damage at } \\
100 \mathrm{~mm}\end{array}$ & $\begin{array}{l}\text { Damage at } \\
1000 \mathrm{~mm}\end{array}$ & & \\
\hline Conventional & -45.9 & 739.0 & 676.1 & 0.580 & 0.369 & 7616 & 0.528 \\
\hline Magnetic mounting & -61.0 & 465.9 & 581.2 & 0.079 & 0.102 & 2393 & 0.653 \\
\hline MWBEMI, $1.0 \mathrm{~mm}$ & -34.5 & 341.6 & 359.7 & 0.078 & 0.097 & 5509 & 0.547 \\
\hline MWBEMI, $0.5 \mathrm{~mm}$ & -36.0 & 124.2 & 129.4 & 0.026 & 0.033 & 4082 & 0.868 \\
\hline
\end{tabular}

PLB: pencil lead break; PSD: power spectral density; EMI: electromechanical impedance; RMSD: root-mean-square deviation; CCDM: correlation coefficient deviation metric; FRF: frequency response function; MWBEMI: metal-wire-based electromechanical impedance.

\section{Conclusion}

This study presents a comparative analysis of the effects of three transducer mounting methods on sensitivity in structural damage detection based on the conventional EMI and transfer FRF methods. The experimental results indicate that the transducer mounting method influences damage detection as well as the frequency bands sensitive to damage. Conventional mounting, in which the transducer is attached directly to the structure using a high-stiffness adhesive, achieves a higher sensitivity to damage. However, the alternative mounting methods can achieve a good sensitivity to damage if the frequency range is properly selected and can provide the advantages of allowing transducer reuse, easy repositioning, and the monitoring of inaccessible structures. Therefore, the results presented in this study can be a good reference for future research using alternative transducer mountings.

\section{Acknowledgements}

The author(s) are grateful to the anonymous reviewers for their valuable recommendations for improving this article.

\section{Declaration of conflicting interests}

The author(s) declared no potential conflicts of interest with respect to the research, authorship, and/or publication of this article.

\section{Funding}

The author(s) disclosed receipt of the following financial support for the research, authorship, and/or publication of this article: This research was supported by São Paulo Research Foundation (FAPESP; Grant No.: 2015/02500-6).

\section{References}

Albakri M and Tarazaga P (2017) Electromechanical impedance-based damage characterization using spectral element method. Journal of Intelligent Material Systems and Structures 28(1): 63-77.
Annamdas VGM and Annamdas KK (2009) Different types of piezoceramic-structure interaction models using electro mechanical impedance technique: a review. In: Proceedings of the SPIE 7292, sensors and smart structures technologies for civil, mechanical, and aerospace systems, San Diego, CA, pp. 1-12. Available at: http://dx.doi.org/10.1117/ 12.817497

Arjun V, Sasi B, Purna B, et al. (2015) Optimisation of pulsed eddy current probe for detection of sub-surface defects in stainless steel plates. Sensors and Actuators A: Physical 226(5): 69-75.

ASTM (2010) Standard Guide for Determining the Reproducibility of Acoustic Emission Sensor Response (ASTM standard E976-10). Available at: http://dx.doi.org/10.1520/ E0976-10

Bakht B and Mufti A (2015) Structural health monitoring. In: Bakht B and Mufti A (eds) Bridges: Analysis, Design, Structural Health Monitoring, and Rehabilitation. Cham: Springer, pp. 307-354.

Baptista FG and Vieira Filho J (2009) A new impedance measurement system for PZT-based structural health monitoring. IEEE Transactions on Instrumentation and Measurement 58(10): 3602-3608.

Baptista FG and Vieira Filho J (2010a) Optimal frequency range selection for PZT transducers in impedance-based SHM systems. IEEE Sensors Journal 10(8): 1297-1303.

Baptista FG and Vieira Filho J (2010b) Transducer loading effect on the performance of PZT-based SHM systems. IEEE Transactions on Ultrasonics, Ferroelectrics, and Frequency Control 57(4): 933-941.

Baptista FG, Budoya DE, de Almeida VAD, et al. (2014) An experimental study on the effect of temperature on piezoelectric sensors for impedance-based structural health monitoring. Sensors 14(1): 1208-1227.

Baptista FG, Vieira Filho J and Inman DJ (2011) Sizing PZT transducers in impedance-based structural health monitoring. IEEE Sensors Journal 11(6): 1405-1414.

Brunner AJ, Birchmeier M, Melnykowycz MM, et al. (2009) Piezoelectric fiber composites as sensor elements for structural health monitoring and adaptive material systems. Journal of Intelligent Material Systems and Structures 20(9): 1045-1055.

Cao H, Thakar SK, Oseng ML, et al. (2015) Development and characterization of a novel interdigitated capacitive 
strain sensor for structural health monitoring. IEEE Sensors Journal 15(11): 6542-6548.

Cortez NE, Vieira Filho J and Baptista FG (2013) A new microcontrolled structural health monitoring system based on the electromechanical impedance principle. Structural Health Monitoring 12(1): 14-22.

De Almeida VAD, Baptista FG and de Aguiar PR (2015) Piezoelectric transducers assessed by the pencil lead break for impedance-based structural health monitoring. IEEE Sensors Journal 15(2): 693-702.

Farrar CR and Worden K (2013) Structural Health Monitoring: A Machine Learning Perspective. Chichester: John Wiley \& Sons.

Freitas ES and Baptista FG (2016) Experimental analysis of the feasibility of low-cost piezoelectric diaphragms in impedance-based SHM applications. Sensors and Actuators A: Physical 238: 220-228.

Giurgiutiu V (2013) Embedded NDT with piezoelectric wafer active sensors. In: Güneş $\mathrm{O}$ and Akkaya Y (eds) Nondestructive Testing of Materials and Structures (RILEM book series, vol. 6). Dordrecht: Springer, pp. 987-992.

Kamas T and Tekkalmaz M (2016) SHM of thick structures via $\mathrm{E} / \mathrm{M}$ impedance of embedded PWAS for weight loss defects. In: Proceedings of the 8th European workshop on structural health monitoring ( EWSHM 2016), Bilbao, 5-8 July.

Kaur N, Bhalla S, Shanker R, et al. (2016) Experimental evaluation of miniature impedance chip for structural health monitoring of prototype steel/RC structures. Experimental Techniques 40(3): 981-992.

Kim I and Chattopadhyay A (2015) Guided Lamb wavebased structural health monitoring using a novel wave packet tracing method for damage localization and size quantification. Journal of Intelligent Material Systems and Structures 26(18): 2515-2530.

Liang C, Sun FP and Rogers CA (1994) Coupled electromechanical analysis of adaptive material systemsdetermination of the actuator power consumption and system energy transfer. Journal of Intelligent Material Systems and Structures 5(1): 12-20.

Martowicz A, Sendecki A, Salamon M, et al. (2016) Application of electromechanical impedance-based SHM for damage detection in bolted pipeline connection. Nondestructive Testing and Evaluation 31(1): 17-44.

Maruo C II, Giachero GF, Steffen Junior V, et al. (2015) Electromechanical impedance-based structural health monitoring instrumentation system applied to aircraft structures and employing a multiplexed sensor array. Journal of Aerospace Technology and Management 7(3): 294-306.

Meitzler AH, Tiersten HF, Berlincourt D, et al. (1988) IEEE Standard on Piezoelectricity: An American National Standard (Standard 176). New York: IEEE-ANSI, 66 pp.

Moharana S and Bhalla S (2015) Influence of adhesive bond layer on power and energy transduction efficiency of piezo-impedance transducer. Journal of Intelligent Material Systems and Structures 26(3): 247-259.

$\mathrm{Na} \mathrm{S}$ and Lee H (2013) Steel wire electromechanical impedance method using a piezoelectric material for composite structures with complex surfaces. Composite Structures 98(4): 79-84.

$\mathrm{Na}$ S, Tawie R and Lee H (2012) Electromechanical impedance method of fiber-reinforced plastic adhesive joints in corrosive environment using a reusable piezoelectric device. Journal of Intelligent Material Systems and Structures 23(7): 737-747.

Na W and Park KT (2017) A cost-effective impedance-based structural health monitoring technique for steel structures by monitoring multiple areas. Journal of Intelligent Material Systems and Structures 28(2): 154-162.

Naskar S and Bhalla S (2016) Metal-wire-based twin onedimensional orthogonal array configuration of PZT patches for damage assessment of two-dimensional structures. Journal of Intelligent Material Systems and Structures 27(11): 1440-1460.

Ohtsu M, Enoki M, Mizutani Y, et al. (2016) Principles of the acoustic emission (AE) method and signal processing. In: Practical Acoustic Emission Testing. Tokyo, Japan: Springer, pp. 5-34. Available at: http://dx.doi.org/10.1007/ 978-4-431-55072-3_2.

Overly TG, Gyuhae P, Farinholt KM, et al. (2009) Piezoelectric active-sensor diagnostics and validation using instantaneous baseline data. IEEE Sensors Journal 9(11): 1414-1421.

Park G, Sohn H, Farrar CR, et al. (2003) Overview of piezoelectric impedance-based health monitoring and path forward. The Shock and Vibration Digest 35(6): 451-463.

Shahab S and Erturk A (2016) Coupling of experimentally validated electroelastic dynamics and mixing rules formulation for macro-fiber composite piezoelectric structures. Journal of Intelligent Material Systems and Structures. Epub ahead of print 3 November. DOI: 10.1177/ 1045389X16672732.

Tinoco HA and Marulanda DJ (2015) Damage identification in active plates with indices based on Gaussian confidence ellipses obtained of the electromechanical admittance. Journal of Nondestructive Evaluation 34: 28.

Vieira Filho J, Baptista FG and Inman DJ (2011) Timedomain analysis of piezoelectric impedance-based structural health monitoring using multilevel wavelet decomposition. Mechanical Systems and Signal Processing 25(5): 1550-1558.

Waugh RC (2016) Non-destructive evaluation. In: Waugh RC (ed.) Development of Infrared Techniques for Practical Defect Identification in Bonded Joints (Springer theses). Cham: Springer, pp. 21-37. Available at: http://dx.doi.org/ 10.1007/978-3-319-22982-9_3

Zhao J, Bao T and Amjad U (2015) Optical fiber sensing of small cracks in isotropic homogeneous materials. Sensors and Actuators A: Physical 225(4): 133-138. 